\title{
Localized Magnetic Impurity States and Magnetic Moments in a Non-Magnetic host metal
}

\author{
AyanaYihunie, Amarendra Rajput, And GetachewAbebe \\ Department of Physics, College of Natural and Computational Sciences Haramaya university, Diredawa-138, \\ Ethiopia \\ Corresponding author :Amarendra Rajput, Department of Physics, Haramaya University, Diredawa- \\ 138,Ethiopia
}

\begin{abstract}
A simplified model Hamiltonian is presented and applied to calculate the density of magnetic impurity states and to study the formation of local magnetic moments in a non-magnetic host metal when a small amount of magnetic impurities is dissolved in it. For this purpose, the many- body Anderson impurity Hamiltonian is transformed into an effective one-body Hamiltonian within the framework of second quantization.This Hamiltonian is then used to derive the equations of motion for the double-time retarded Green's functions in Zubarev's notation. These Green's functions are employed to calculate the density of impurity states and the average occupation number of impurity electrons with specific spin orientations. Finally the general magnetic solutions are found and a phase diagram is drawn to show how local magnetic moments may be formed under suitable conditions determined by an intricate interplay of certain physical parameters such as impurity energy levels, on-site coulomb repulsion and the hybridization energy.
\end{abstract}

Keywords: - Anderson impurity Model, second quantization, double-time Green's function, hybridization energy, density of states.

\section{INTRODUCTION}

One of the most challenging problems in condensed matter physics has been to understand the properties of magnetic impurities in a non-magnetic host metal. It was as early as 1930's, when anomalies in the low-temperature resistivity of the host metalwere experimentally discovered [1]. The origin of this anomaly was later successfully explained by Kondo [2] in terms of spin-exchange interactions between impurity and itinerant electrons. Again, around 1960's, in a series of ESR and NMR experiments on metals, Matthias et. al. [3] found surprising evidence of the existence of local magnetic moments, which were soon attributed to the presence of small amounts of magnetic impurities. Not only that, later experiments by Riegel and Gross [4] and Kapoor et.al. [5]brought out some more intriguing results. It was found that iron impurities in Group V metals $\mathrm{V}, \mathrm{Nb}$, and Ta showed zero magnetic moments, whereas in Group VI metals $\mathrm{Cr}$, Mo and $\mathrm{W}$, there existed nonzero localized magnetic moments. Recently some new experimental techniques [6] have been developed in this direction.

These exciting experimental discoveries led to a spar of theoretical activities to understand the mechanism of formation of localized magnetic moments. The first pioneering work in this direction was initiated by Anderson [7] following the ideas proposed by Friedel [8]. Anderson's model Hamiltonian has been a milestone in understanding the properties of magnet impurities, including the Kondo physics. It still serves as a foundation for various more refined techniques used by later workers $[9,10]$. The specific problem of magnetic impurity on graphene has recently been studied by several groups [11-14]. A critical analysis of the Friedel-Anderson model has been made by Bergmann [15]. The existence and stability of local magnetic moments in non-magnetic metals has proved to be very crucial for our understanding of many physical phenomena, such as giant local moments, spin glasses [16] and magnetic ordering in some concentrated alloys and compounds [17].

In this paper, we consider a host metal with an s-band, in which a transition metal is dissolved. -We assume that the s-electrons can hop into the d-shell valance electrons of the impurity atom via the hybridization matrix element. The ten-fold degeneracy of a real d-impurity is simplified to a two-fold degeneracy of spin up and spin down. Employing the equivalent one-body Anderson Hamiltonian and the techniques of retarded double-time Green's functions, we explore the properties of the magnetic impurity. We then obtain a phase diagram which shows clearly why for certain values of the physical parameters, it is energetically favourable for the system to have a local magnetic moment, while, for others magnetic moment does not exists. 
A. Model Hamiltonian

\section{METHOD OF CALCULATION}

The general Hamiltonian in the second-quantized form is given by

$$
H=\int d^{3} r \psi^{+}\left(-\frac{\hbar^{2}}{2 m} \nabla^{2}+U\right) \psi
$$

where $\psi$ and $\psi^{+}$are the field operators.

In the present problem, we have to take into account both a localized d-impurity electron with an orbital $\phi_{d}(r)$ and conduction electrons, each with a wave function $\phi_{k}(r)$. In that case, the field operators take the following general forms.

$$
\psi(r)=\sum_{k \sigma} \phi_{k}(r) c_{k \sigma}+\sum_{\sigma} \phi_{d}(r) c_{d \sigma}(2)
$$

$\operatorname{and} \psi(r)^{+}=\sum_{k \sigma} \phi_{k}(r)^{*} c_{k \sigma}{ }^{+}+\sum_{\sigma} \phi_{d}(r)^{*} c_{d \sigma}{ }^{+}(3)$

wherec $c_{k \sigma}\left(c_{k \sigma}{ }^{+}\right)$and $c_{d \sigma}\left(c_{k \sigma}{ }^{+}\right)$are annihilation(creation) operators for a conduction and impurity electron of spin $\sigma$, respectively.

Inserting (2) and (3) in (1), and after some calculation, one arrives at the following Hamiltonian.

$H=\sum_{k \sigma} \epsilon_{k} c_{k \sigma}{ }^{+} c_{k \sigma}+\sum_{\sigma} \epsilon_{d} c_{d \sigma}{ }^{+} c_{d \sigma}+\sum_{k \sigma} V_{k d}\left(c_{k \sigma}{ }^{+} c_{d \sigma}+c_{d \sigma}{ }^{+} c_{k \sigma}\right)+\sum_{k \sigma} U_{k} c_{k \sigma}{ }^{+} c_{k \sigma}$

In (4), $\epsilon_{k}$ and $\epsilon_{d}$ are single-particle energies for conduction and impurity electronsrespectively. $V_{k d}$ is the hybridization (called s-d interaction) term and $U_{k}$ is the potential for scattering of conduction electrons.

In the study of magnetic moments, the term containing $U_{k}$ plays no role and can be ignored. Again if the impurity d-state is non-degenerate, then the orbital can be either singly occupied or occupied by electrons of opposite spins. The latter case costs an energy $U$ which arises from the coulomb repulsion between the two delectrons, and this given by,

$U=\int d^{3} r_{1} d^{3} r_{2}\left|\phi_{d}\left(r_{1}\right)\right|^{2} \frac{e^{2}}{\left|r_{1}-r_{2}\right|}\left|\phi_{d}\left(r_{2}\right)\right|^{2}$

This interaction, in second quantized form, will be given by

$U c_{d \sigma}{ }^{+} c_{d \sigma^{\prime}}{ }^{+} c_{d \sigma^{\prime \prime}} c_{d \sigma^{\prime \prime \prime}}$

Since, there are only two spin directions (up or down), we follow Anderson [7] and replace (6) by

$U n_{d \sigma} n_{d-\sigma}$

$$
\text { where } n_{d \sigma}=c_{d \sigma}^{+} c_{d \sigma}
$$

and $n_{d-\sigma}=c_{d-\sigma}^{+} c_{d-\sigma}$ are the number operators for d-electrons of opposite spins.

The expression (7) is called the correlation energy of the localized d-electrons. Introducing (7) in (4), we the have

$H=\sum_{k \sigma} \epsilon_{k} c_{k \sigma}{ }^{+} c_{k \sigma}+\sum_{\sigma} \epsilon_{d} c_{d \sigma}{ }^{+} c_{d \sigma}+\sum_{k \sigma} V_{k d}\left(c_{k \sigma}{ }^{+} c_{d \sigma}+c_{d \sigma}{ }^{+} c_{k \sigma}\right)+U n_{d \sigma} n_{d-\sigma}$

This is exactly identical with the Anderson Hamiltonian, which can be transformed into an effective one-body Hamiltonian so that mathematical complexities may be reduced to the minimum without compromising the underlying physics.

We write the ground state of the system as

$\left|\phi_{0}\right\rangle=\prod_{\epsilon_{n}<\epsilon_{F}} c_{n \sigma}^{+}|0\rangle$

wherec $_{n \sigma}^{+}=\sum_{k}\langle n \mid k\rangle_{\sigma} c_{k \sigma}^{+}+\langle n \mid d\rangle_{\sigma} c_{d \sigma}^{+}$

is a linear combination of band and impurity states.

Taking averages in the state $\left|\phi_{0}\right\rangle$, we now replace the interaction term (7) as follows;

$U n_{d \sigma} n_{d-\sigma} \rightarrow U\left\langle n_{d \sigma}\right\rangle n_{d-\sigma}+U\left\langle n_{d-\sigma}\right\rangle n_{d \sigma}-U\left\langle n_{d \sigma}\right\rangle\left\langle n_{d-\sigma}\right\rangle$

The last term in (11) provides an overall shift in zero of energy. Using (11) in (8), we obtain

$H=$

$\sum_{k \sigma} \epsilon_{k} c_{k \sigma}{ }^{+} c_{k \sigma}+\sum_{\sigma} \epsilon_{d} c_{d \sigma}{ }^{+} c_{d \sigma}+\sum_{k \sigma} V_{k d}\left(c_{k \sigma}{ }^{+} c_{d \sigma}+c_{d \sigma}{ }^{+} c_{k \sigma}+U\left\langle c_{d \sigma}^{+} c_{d \sigma}\right\rangle c_{d-\sigma}^{+} c_{d-\sigma}+\right.$

$U\left\langle c_{d-\sigma}^{+} c_{d-\sigma}\right\rangle c_{d \sigma}^{+} c_{d \sigma}-U\left\langle c_{d \sigma}^{+} c_{d \sigma}\right\rangle\left\langle c_{d-\sigma}^{+} c_{d-\sigma}\right\rangle$ (12)

To simplify the Hamiltonian in (12), we neglect the correlations between up and down spins in the impurity orbital. Considering the fluctuations of the down spin to be small, we can write the spin-down number operator as

$c_{d-\sigma}^{+} c_{d-\sigma}=\left(c_{d-\sigma}^{+} c_{d-\sigma}-\left\langle c_{d-\sigma}^{+} c_{d-\sigma}\right\rangle\right)+\left\langle c_{d-\sigma}^{+} c_{d-\sigma}\right\rangle \approx\left\langle c_{d-\sigma}^{+} c_{d-\sigma}\right\rangle \equiv\left\langle n_{d-\sigma}\right\rangle$

Where we have used the fact that the deviation of number operator from the mean is negligible. Using (13) in (12) we have

$U\left\langle c_{d-\sigma}^{+} c_{d-\sigma}\right\rangle c_{d \sigma}^{+} c_{d \sigma}$

$$
H=\sum_{k \sigma} \epsilon_{k} c_{k \sigma}{ }^{+} c_{k \sigma}+\sum_{\sigma} \epsilon_{d} c_{d \sigma}{ }^{+} c_{d \sigma}+\sum_{k \sigma} V_{k d}\left(c_{k \sigma}{ }^{+} c_{d \sigma}+c_{d \sigma}{ }^{+} c_{k \sigma}\right)+
$$

Defining the defect site energy as

$E_{d \sigma}=\epsilon_{d}+U\left\langle c_{d-\sigma}^{+} c_{d-\sigma}\right\rangle$

We write (14) as

$H_{e f f}=\sum_{k \sigma} \epsilon_{k} c_{k \sigma}^{+} c_{k \sigma}+\sum_{\sigma} E_{d \sigma} c_{d \sigma}^{+} c_{d \sigma}+\sum_{k \sigma}\left(V_{k d} c_{k \sigma}^{+} c_{d \sigma}+V_{d k} c_{d \sigma}^{+} c_{k \sigma}\right)$

Equation (16) is the desired one-body Hamiltonian which will be utilized in the following calculations. 


\section{B. Equations of Motion for Green's Function and Density of Impurity States}

We use the temperature dependent retarded Green function in Zubarev notation [18], given by

$G_{A B}^{r}\left(t-t^{\prime}\right) \equiv\left\langle\left\langle A(t) ; B\left(t^{\prime}\right)\right\rangle\right\rangle \equiv-i \theta\left(t-t^{\prime}\right)\left\langle[A, B]_{+}\right\rangle$

Where $\mathrm{A}(\mathrm{t})$ and $\mathrm{B}\left(\mathrm{t}^{\prime}\right)$ are the Heisenberg operators and $\theta\left(t-t^{\prime}\right)$ is the Heaviside function. The time derivative of Heaviside step function is given by Dirac delta function,

$\frac{d}{d t} \theta\left(t-t^{\prime}\right)=\delta\left(t-t^{\prime}\right)$

Where $\theta\left(t-t^{\prime}\right)= \begin{cases}1 & \text { if } t>t^{\prime} \\ 0 & \text { if } t<t^{\prime}\end{cases}$

Since $\mathrm{B}\left(\mathrm{t}^{\prime}\right)$ is independent of $\mathrm{t}$, the time - derivative of (17) will be given by

$i \frac{d}{d t} G_{A B}{ }^{r}\left(t-t^{\prime}\right)=\delta\left(t-t^{\prime}\right)\left\langle[A, B]_{+}\right\rangle+\theta\left(t-t^{\prime}\right)\left\langle\left[\frac{d}{d t} A, B\right]_{+}\right\rangle$

The Heisenberg equation for $A(\mathrm{t})$ is given by;

$i \hbar \frac{d}{d t} A=[A(t), H]$

Using (21) in (20) (with $\hbar=1$ ) and then the definition (17), we get

$i \frac{d}{d t} G_{A B}{ }^{r}\left(t-t^{\prime}\right)=\delta\left(t-t^{\prime}\right)\left\langle[A, B]_{+}\right\rangle+\ll[A(t), H] ; B\left(t^{\prime}\right) \gg$

Since the Fourier transform of $\delta\left(t-t^{\prime}\right)$ is1, we can easily obtain the Fourier transform of $G_{A B}{ }^{r}\left(t-t^{\prime}\right)$ as follows. Thus

$G_{A B}^{r}\left(t-t^{\prime}\right)=\int_{-\infty}^{\infty} G_{A B}^{r}(\omega) \exp \left(r-i \omega\left(t-t^{\prime}\right) d \omega\right.$

So that, Fourier transform of $\frac{d}{d t} G_{A B}{ }^{r}\left(t-t^{\prime}\right)=(-i \omega) G_{A B}{ }^{r}(\omega)$

Taking the Fourier transform of the entire equation (22) and using (23), we obtain

$\omega G_{A B}{ }^{r}(\omega)=\left\langle[A, B]_{+}\right\rangle+\left\langle\langle[A, H] ; B\rangle_{\omega}\right.$

Using (17) and (25), we can write the following equation of motion for the Green function of the creation and annihilation operators for localized electrons.

$\omega \ll c_{d \sigma} ; c_{d \sigma}^{+} \gg=\left\langle\left[c_{d \sigma}, c_{d \sigma}^{+}\right]_{+}\right\rangle+\left\langle\left[c_{d \sigma}, H_{e f f}\right]_{-} ; c_{d \sigma}^{+}\right\rangle_{\omega}$

where $H_{\text {eff }}$ is given by (17). Using the anti-commutation relations for fermion operators andafter some lengthy but straight forward calculations, one obtains,

$\left[c_{d \sigma}, H_{e f f}\right]_{-}=\sum_{\sigma^{\prime}} E_{d \sigma^{\prime}} c_{d \sigma^{\prime}} \delta_{\sigma \sigma^{\prime}}+\sum_{k \sigma^{\prime}} V_{d k} c_{k \sigma^{\prime}} \delta_{\sigma \sigma^{\prime}}=E_{d \sigma} c_{d \sigma}+\sum_{k \sigma} V_{d k} c_{k \sigma}$

Substituting (27) in (26), we get,

$\omega\left\langle c_{d \sigma} ; c_{d \sigma}^{+} \gg=1+E_{d \sigma}\left\langle c_{d \sigma} ; c_{d \sigma}^{+} \gg+\sum_{k \sigma} V_{d k}\left\langle\left\langle c_{k \sigma} ; c_{d \sigma}^{+} \gg\right.\right.\right.\right.$

Next we calculate the Green's function $\left\langle c_{d \sigma} ; c_{k \sigma}^{+} \gg\right.$. Its equation of motion is given by

$\omega\left\langle c_{d \sigma} ; c_{k \sigma}^{+} \gg=\left\langle\left[c_{d \sigma}, c_{k \sigma}^{+}\right]_{+}\right\rangle+\left\langle\left\langle c_{d \sigma}, H_{e f f}\right]_{-} ; c_{k \sigma}^{+}\right\rangle_{\omega}\right.$

where $\left[c_{d \sigma}, c_{k \sigma}^{+}\right]_{+}=0$

Following similar calculations, we arrive at the following result.

$\left[c_{d \sigma}, H_{e f f}\right]_{-}=\sum_{\sigma^{\prime}} E_{d \sigma^{\prime}} c_{d \sigma^{\prime}} \delta_{\sigma \sigma^{\prime}}+\sum_{k \sigma^{\prime}} V_{d k} c_{k \sigma^{\prime}} \delta_{\sigma \sigma^{\prime}}=E_{d \sigma} c_{d \sigma}+\sum_{k \sigma} V_{d k} c_{k \sigma}$

Using (30) and (31) in (29), we get

$\omega\left\langle c_{d \sigma} ; c_{k \sigma}^{+} \gg=E_{d \sigma}\left\langle\left\langle c_{d \sigma} ; c_{k \sigma}^{+} \gg+\sum_{k \sigma} V_{d k}\left\langle\left\langle c_{k \sigma} ; c_{k \sigma}^{+} \gg\right.\right.\right.\right.\right.$

Similarly the Green function $\left\langle\left\langle c_{k \sigma} ; c_{k \sigma}^{+} \gg\right.\right.$ is given by,

$\left.\omega\left\langle c_{k \sigma} ; c_{k \sigma}^{+}\right\rangle=\left\langle\left[c_{k \sigma}, c_{k \sigma}^{+}\right]_{+}\right\rangle+\ll\left[c_{k \sigma}, H_{e f f}\right]_{-} ; c_{k \sigma}^{+}\right\rangle_{\omega}$

Using the anti-commutation relation,

$\left[c_{k \sigma}, c_{k^{\prime} \sigma^{\prime}}^{+}\right]_{+}=\delta_{k k^{\prime}} \delta_{\sigma \sigma^{\prime}}$

one can arrive at the result

$\left[c_{k \sigma}, H_{e f f}\right]_{-}=\sum_{k^{\prime} \sigma^{\prime}} \epsilon_{k^{\prime}} c_{k^{\prime} \sigma}, \delta_{\sigma \sigma^{\prime}}, \delta_{k k^{\prime}}+\sum_{k^{\prime} \sigma^{\prime}} V_{k^{\prime} d} c_{d \sigma^{\prime}} \delta_{\sigma \sigma^{\prime}}, \delta_{k k^{\prime}}$

Using (34) and (35) in (33), we get

$\omega\left\langle c_{k \sigma} ; c_{k \sigma}^{+} \gg=1+\epsilon_{k}\left\langle\left\langle c_{k \sigma} ; c_{k \sigma}^{+} \gg+V_{k d}\left\langle\left\langle c_{d \sigma} ; c_{k \sigma}^{+} \gg\right.\right.\right.\right.\right.$

Again, the Green function $\left\langle c_{k \sigma} ; c_{d \sigma}^{+} \gg\right.$ is given by

$\left.\omega\left\langle c_{k \sigma} ; c_{d \sigma}^{+}\right\rangle=\left\langle\left[c_{k \sigma}, c_{d \sigma}^{+}\right]_{+}\right\rangle+\ll\left[c_{k \sigma}, H_{e f f}\right]_{-} ; c_{d \sigma}^{+}\right\rangle_{\omega}$

where,

$\left[c_{k \sigma}, c_{d \sigma}^{+}\right]_{+}=0$

It then follows that

$\omega\left\langle\left\langle c_{k \sigma} ; c_{d \sigma}^{+} \gg=\epsilon_{k}\left\langle\left\langle c_{k \sigma} ; c_{d \sigma}^{+} \gg+V_{k d}\left\langle\left\langle c_{d \sigma} ; c_{d \sigma}^{+} \gg\right.\right.\right.\right.\right.\right.$

We now collect all the equations of motions for the above Green's functions. Then, from (28), we have

From (32), 
$\left\langle c_{d \sigma} ; c_{k \sigma}^{+} \gg=\sum_{k \sigma} V_{d k} \frac{\ll c_{k \sigma} ; c_{k \sigma}^{+} 》}{\left(\omega-E_{d \sigma}\right)}\right.$

From (36),

$\ll c_{k \sigma} ; c_{k \sigma}^{+} \gg=\frac{1}{\left(\omega-\epsilon_{k}\right)}+V_{k d} \frac{\ll c_{d \sigma} ; c_{k \sigma}^{+} 》}{\left(\omega-\epsilon_{k}\right)}$

And from (39),

$\left\langle c_{k \sigma} ; c_{d \sigma}^{+} \gg=V_{k d} \frac{\left\langle\left\langle c_{d \sigma} ; c_{d \sigma}^{+} \gg\right.\right.}{\left(\omega-\epsilon_{k}\right)}\right.$

For convenience, we use the following abbreviations for Green's functions with spin up index

$G_{d d}^{\sigma}=\left\langle\left\langle c_{d \sigma} ; c_{d \sigma}^{+} \gg\right.\right.$

$G_{d k}^{\sigma}=\left\langle\left\langle c_{d \sigma} ; c_{k \sigma}^{+} \gg\right.\right.$

$G_{k k}^{\sigma}=\left\langle\left\langle c_{k \sigma} ; c_{k \sigma}^{+} \gg\right.\right.$

$G_{k d}^{\sigma}=\left\langle\left\langle c_{k \sigma} ; c_{d \sigma}^{+} \gg\right.\right.$

To calculate the density of states for the localized electrons, we now employ the equation of motion for the Green function of the impurity level.Using (43) in (40) and the abbreviated notation in(44), we get

It then follows that

$$
\left(\omega-E_{d \sigma}\right) G_{d d}^{\sigma}=1+\sum_{k \sigma} V_{d k} V_{k d}\left(\frac{1}{\omega-\epsilon_{k}}\right) G_{d d}^{\sigma}=1+\sum_{k \sigma}\left|V_{d k}\right|^{2}\left(\frac{1}{\omega-\epsilon_{k}}\right) G_{d d}^{\sigma}
$$

$G_{d d}^{\sigma}=\left[\omega-E_{d \sigma}-\sum_{k \sigma} \frac{\left|V_{d k}\right|^{2}}{\left(\omega-\epsilon_{k}\right)}\right]^{-1}$

Writing $\epsilon=\hbar \omega$ and $\hbar=1$,we can rewrite (45) as

$G_{d d}^{\sigma}(\epsilon+i \eta)=\left[\epsilon+i \eta-E_{d \sigma}-\sum_{k \sigma} \frac{\left|V_{d k}\right|^{2}}{\left(\epsilon-\epsilon_{k}+i \eta\right)}\right]^{-1}$

where $G_{d d}^{\sigma}(\epsilon+i \eta)$ is the diagonal d- orbital Green function with spin up and down. $E_{d \sigma}$ is the defect site energy given by

$E_{d \sigma}=\epsilon_{d}+U\left\langle n_{d,-\sigma}\right\rangle$.

The positive constant $\eta$ is introduced to take care of singularities. Using the mathematical result[19] $\lim _{\eta \rightarrow 0} \frac{1}{x+i \eta}=\wp\left(\frac{1}{x}\right)-i \pi \delta(x)$

Where $\wp$ denotes the principal value, we have

$\lim _{\eta \rightarrow 0} \sum_{k \sigma} \frac{\left|V_{d k}\right|^{2}}{\epsilon-\epsilon_{k}+i \eta}=\wp\left[\sum_{k \sigma} \frac{\left|V_{d k}\right|^{2}}{\epsilon-\epsilon_{k}}\right]-i \pi \sum_{k \sigma}\left|V_{d k}\right|^{2} \delta\left(\epsilon-\epsilon_{k}\right)$

The first term on the right of (49) is purely real and hence, represents a shift of the d-impurity energy level. This term, therefore, does not affect the physics and can be ignored. Then (49) gives

$\lim _{\eta \rightarrow 0} \sum_{k \sigma} \frac{\left|V_{d k}\right|^{2}}{\epsilon-\epsilon_{k}+i \eta}=-i \pi \sum_{k}\left|V_{d k}\right|^{2} \delta\left(\epsilon-\epsilon_{k}\right)$

We now put $\quad \Delta=\pi \sum_{k}\left|V_{d k}\right|^{2} \delta\left(\epsilon-\epsilon_{k}\right)$

This represents the effective transition rate between the impurity and the conduction electrons. Physically $\Delta$ is the width of the broadening of the impurity energy levels and can be assumed to be constant, independent of $E_{d \sigma}$. Using (50) and (51) in (46), we then get

$G_{d d}^{\sigma}(\epsilon+i \eta)=\frac{1}{\epsilon+i \eta-E_{d \sigma}+i \Delta}$

Using (48), we have from (52),

$$
\begin{aligned}
\lim _{\eta \rightarrow 0} G(\epsilon+i \eta)= & \lim _{\eta \rightarrow 0}\left(\frac{1}{\epsilon-E_{d \sigma}+i(\Delta+\eta)}\right) \\
& =p\left(\frac{1}{\epsilon-E_{d \sigma}}\right)-i \pi \delta\left(\epsilon-\epsilon_{d}\right)
\end{aligned}
$$

But the density of states at the d- impurity $\rho_{d \sigma}(\epsilon)$ must be peaked around $E_{d \sigma}$. Then it can be written as $\rho_{d \sigma}(\epsilon)=\delta\left(\epsilon-\epsilon_{d \sigma}\right)$

From (53) and (54), it then follows that

$\rho_{d \sigma d \sigma}(\epsilon)=-\frac{1}{\pi} \lim _{\eta \rightarrow 0} \operatorname{Im} G_{d d}^{\sigma}(\epsilon+i \eta)$

Now from (52), $\quad \operatorname{Im} G_{d d}^{\sigma}(\epsilon+i \eta)=\operatorname{Im}\left(\frac{1}{\epsilon+i \eta-E_{d \sigma}+i \Delta}\right)=-\frac{(\Delta+\eta)}{\left(\epsilon-E_{d \sigma}\right)^{2}+(\Delta+\eta)^{2}}$

Thus $\quad \lim _{\eta \rightarrow 0} I m G_{d d}^{\sigma}(\epsilon+i \eta)=-\frac{\Delta}{\left(\epsilon-E_{d \sigma}\right)^{2}+\Delta^{2}}$

From (55) and (57), we then have, $\quad \rho_{d \sigma}(\epsilon)=\frac{1}{\pi} \frac{\Delta}{\left(\epsilon-E_{d \sigma}\right)^{2}+\Delta^{2}}$

Eqn. (58) give the density of states at the d- impurity and is of the Lorentzian form. As expected, the hybridization energy $\Delta$ appears as the half-width of the d-level. If $\Delta \rightarrow 0$, then $\rho_{d \sigma}(\epsilon)$ behaves like a delta function, with peak at $\epsilon=E_{d \sigma}$. This is shown in Fig.1 and Fig.2. 


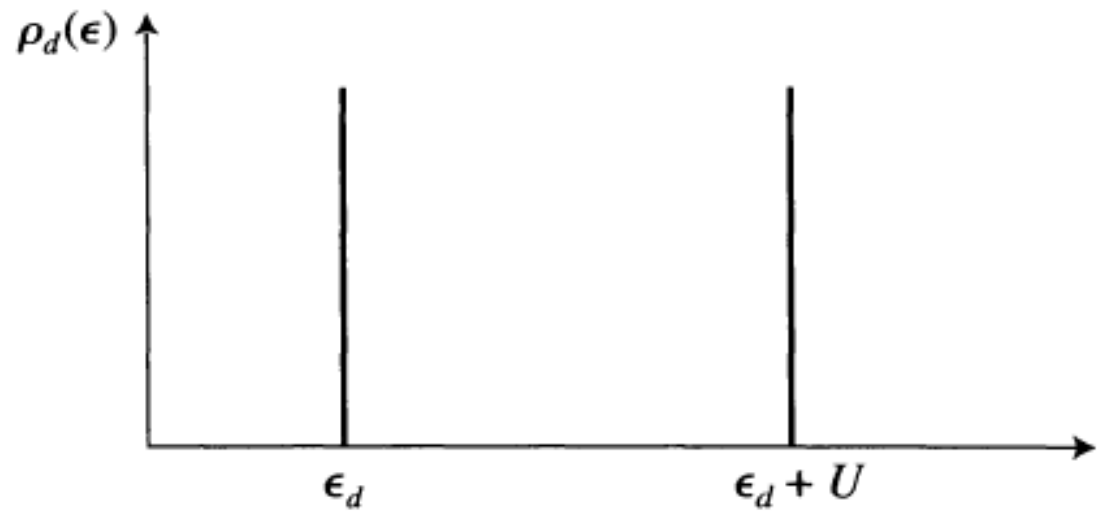

Fig.1. Density of Impurity states when $\Delta \rightarrow \mathbf{0}$

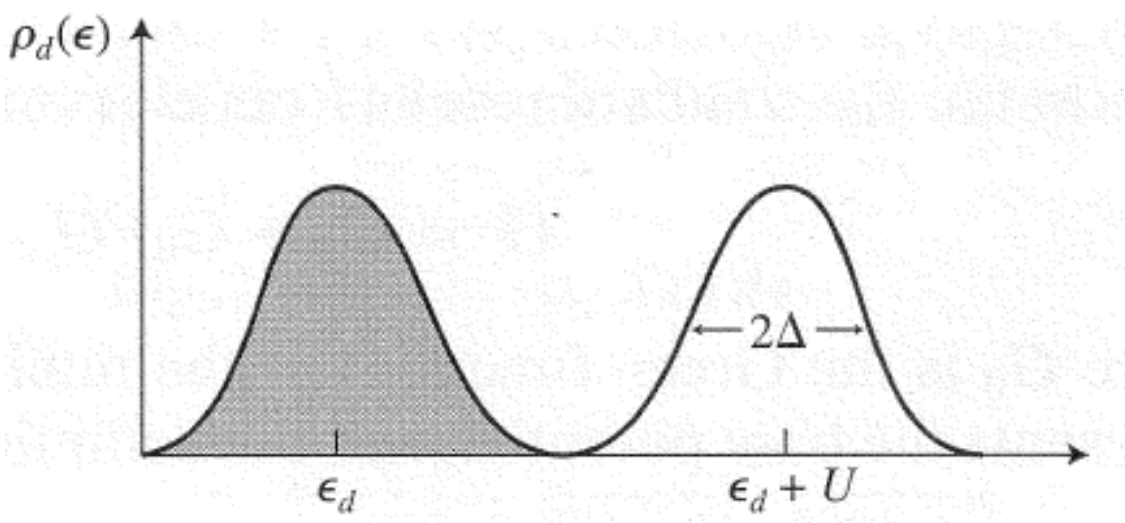

Fig.2. Density of impurity states when d-and k-states are mixed $(\Delta \neq 0)$.

\section{Average Occupation Number of Impurity States}

The average occupation number of d-electrons of a given spin $\sigma$ is obtained by integrating (58) in the continuum energy up to the Fermi level $\epsilon_{F}$, since all states below the Fermi level are full. Taking the Fermi level at zero energy, we have,

$$
\left\langle n_{d \sigma}\right\rangle=\int_{-\infty}^{\epsilon_{F}=0} \rho_{d \sigma}(\epsilon) d \epsilon
$$

$=\frac{\Delta}{\pi} \int_{-\infty}^{\epsilon_{F}=0} \frac{1}{\left(\epsilon-E_{d \sigma}\right)^{2}+\Delta^{2}} d \epsilon$

This can be easily integrated to give

$\left\langle n_{d \sigma}\right\rangle=\frac{1}{\pi}\left\{\tan ^{-1}\left(-\frac{E_{d \sigma}}{\Delta}\right)+\frac{\pi}{2}\right\}$

Since, $\tan ^{-1}(-\mathrm{x})=-\tan ^{-1}(\mathrm{x})$ we have

$\left\langle n_{d \sigma}\right\rangle=\frac{1}{\pi}\left\{\frac{\pi}{2}-\tan ^{-1}\left(\frac{E_{d \sigma}}{\Delta}\right)\right\}$

Again, $\quad \frac{\pi}{2}-\tan ^{-1} y=\cot ^{-1} y$

Using (61) and (47) in (60), we have finally,

$\left\langle n_{d \sigma}\right\rangle=\frac{1}{\pi} \cot ^{-1}\left(\frac{\epsilon_{d}+U\left\langle n_{d,-\sigma}\right\rangle}{\Delta}\right)$

It is to be observed that in (62), the spin index appears with opposite signs. This means, for self-consistent solutions, the following two simultaneous equations must hold.

$\left\langle n_{d, \sigma}\right\rangle=\frac{1}{\pi} \cot ^{-1}\left(\frac{\epsilon_{d}+U\left\langle n_{d,-\sigma}\right\rangle}{\Delta}\right)$

$\left\langle n_{d,-\sigma}\right\rangle=\frac{1}{\pi} \cot ^{-1}\left(\frac{\epsilon_{d}+U\left\langle n_{d, \sigma}\right\rangle}{\Delta}\right)$

These two coupled equations give us the occupation of the spin up and spin down states of the impurity. They are the fundamental equations resulting from our simplified Hamiltonian and help us in understanding the conditions for the formation of magnetic moments. 


\section{MAGNETIC AND NON- MAGNETIC SOLUTIONS} AND THE PHASE DIAGRAM

The energies of the available single particle states on the impurity are $\epsilon_{d}$ if the level is unoccupied and $\left(\epsilon_{d}+U\right)$, if the level is already singly occupied. If $U \gg \epsilon_{d} \gg \Delta$, the cost of putting two electrons on the d-level of , the impurity, far exceeds $\epsilon_{d}$ and $\Delta$. In this limit, the system should support local moment formation. Again, $\epsilon_{d}$ should lie below the Fermi level, which implies $\epsilon_{d}<0$.

The energy cost of putting two electrons on the impurity is $2 \epsilon_{d}+U$. If $\left(2 \epsilon_{d}+U\right)>0$, the upper state of the impurity will be unoccupied and the impurity would be magnetic.

If $U \gg \Delta \gg \epsilon_{d}$, the occupation of the lower state undergoes rapid fluctuations due to the mixing of d- and kstates. This situation corresponds to non-magnetic behavior.

Again, if $U \ll \Delta$, it is $\Delta$, which mainly determines the physics. In that case, the impurity has equal probability of being occupied by a spin up or spin-down electron. This state is again non-magnetic. For formation of local moments, we must seek solutions corresponding to the case

Now, when $\Delta \rightarrow \infty$, we have from (63) and (64),

$$
\left\langle n_{d \sigma}\right\rangle \neq\left\langle n_{d-\sigma}\right\rangle
$$

Which

$$
\begin{gathered}
\cot \left(\pi\left\langle n_{d \sigma}\right\rangle\right)=\cot \left(\pi\left\langle n_{d-\sigma}\right\rangle\right)=0 \\
\text { implies }
\end{gathered}
$$

This is again a non-magnetic solution.

$$
\left\langle n_{d \sigma}\right\rangle=\left\langle n_{d-\sigma}\right\rangle=\frac{1}{2}
$$

Again if $U=0$, it follows from (63) and (64) that

This is again a non-magnetic situation.

$$
\left\langle n_{d \sigma}\right\rangle=\left\langle n_{d-\sigma}\right\rangle
$$

Thus weconclude that, a magnetic solution exist only if

This means $y=\frac{U}{\Delta}>>1$

$$
U \rightarrow \text { large, and } \Delta \rightarrow \text { small }
$$

$$
\text { Let us write, } x=\frac{-\epsilon_{d}}{U} \geq 0 \quad\left(\because \epsilon_{d} \leq 0\right)
$$

To determine the possible range of $\mathrm{x}$, we note that, for local moment formation, the d-level energies must satisfy

$\epsilon_{d} \leq 0$ and $\epsilon_{d}+U \geq 0$

The limiting cases of (67) are:

$\epsilon_{d}=0 ; \epsilon_{d}+u=0$

Using (68) in (66), it is clear that the limits on $\mathrm{x}$ are,

$0 \leq x \leq 1$

Thus, the energies $\epsilon_{d}=0$ and $\epsilon_{d}=-U$ are symmetrically located around

$x=\frac{1}{2} \quad$ or $\quad \epsilon_{d}=-\frac{U}{2}$

Hence, $x=\frac{1}{2}$ is the most favorable condition for a magnetic moment to form. We now apply the condition (65), (69) and (70) to (63) and (64) to determine the magneticsolutions. We rewrite the Equation (63) as follows.

Using (65) and (66) in this equations, we have

$$
\cot \pi\left\langle n_{d, \sigma}\right\rangle=\frac{U}{\Delta}\left\{\left\langle n_{d,-\sigma}\right\rangle+\frac{\epsilon_{d}}{U}\right\}
$$

$\cot \pi\left\langle n_{d, \sigma}\right\rangle=y\left(\left\langle n_{d,-\sigma}\right\rangle-x\right)$

Or,

$\tan \pi\left\langle n_{d, \sigma}\right\rangle=\frac{1}{y\left(\left\langle n_{d,-\sigma}\right\rangle-x\right)}$

Writing

$\mathrm{z}=\frac{1}{y\left(\left\langle n_{d,-\sigma}\right\rangle-x\right)}$

We have

$\tan ^{-1} z=\pi\left\langle n_{d \sigma}\right\rangle$

Since y large means z small, we have from (74)

$$
\mathrm{Z}=\pi\left\langle n_{d \sigma}\right\rangle
$$

Again, since, $\tan \theta=\tan (\theta \pm \pi)$, another possible solution of (72) is

$$
\mathrm{Z}=\pi\left\langle n_{d \sigma}\right\rangle \pm \pi
$$

Equation (75) and (76) gives us the following two possibilities.

$\pi\left\langle n_{d, \sigma}\right\rangle=\frac{1}{y\left(\left\langle n_{d,-\sigma}\right\rangle-x\right)}$

and 
$\pi\left\langle n_{d, \sigma}\right\rangle \pm \pi=\frac{1}{y\left(\left\langle n_{d,-\sigma}\right\rangle-x\right)}$

Now for magnetic moment formation, we must have

$\left\langle n_{d, \sigma}\right\rangle>\left\langle n_{d,-\sigma}\right\rangle$.

This means, we must choose in

$\pi\left\langle n_{d \sigma}\right\rangle=\pi+\frac{1}{y\left(\left\langle n_{d,-\sigma}\right\rangle-x\right)}$

And in that case, (77) will give

$\pi\left\langle n_{d,-\sigma}\right\rangle=\frac{1}{y\left(\left(n_{d, \sigma}\right\rangle-x\right)}$

These are consistent as spin indices must have different signs on both sides of the equations.

To draw the phase diagram, we first determine the equation which determines the phase boundary which is defined by

$\left\langle n_{d, \sigma}\right\rangle=\left\langle n_{d,-\sigma}\right\rangle=n_{c}$, Say

Eqn(63) and (64) then reduce to a single equation given by,

$\cot \left(\pi n_{c}\right)=y\left(n_{c}-x\right)$

Taking its derivative with respect to $n_{c}$, we get

$\frac{\pi}{y}=\sin i\left(\pi n_{c}\right)$

Using (83) coupled with the condition (69) for $\mathrm{x}$, we can now draw phase diagram. Using the matlab, the following phase diagram in fig. (3) is obtained.

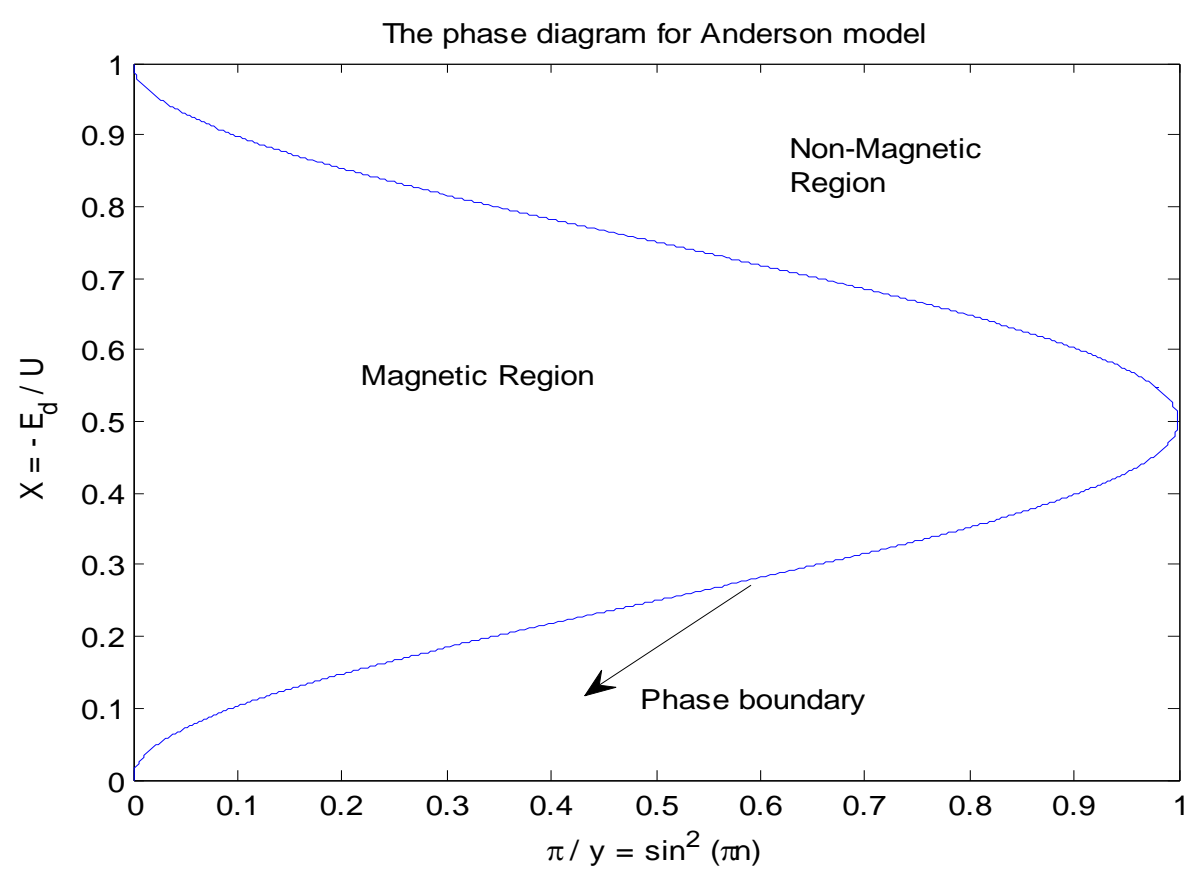

Fig. (3) Phase diagram showing clearly the magnetic and non-magnetic regions. The solid curve is the phase boundary.

We can derive another physical condition related to the phase boundary by Combining (82) and (83). Thus from (82) we have,

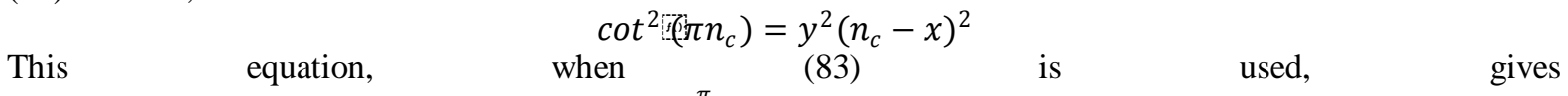

Or

$$
\frac{1-\frac{\pi}{y}}{\frac{\pi}{y}}=y^{2}\left(n_{c}-x\right)^{2}
$$

$\frac{y}{\pi}=1+y^{2}\left(n_{c}-x\right)^{2}=1+\left(y n_{c}-x y\right)^{2}$

Using (65) and (66) in (84), we have

$\frac{U}{\pi \Delta}=1+\left(\frac{\epsilon_{d}+U n_{c}}{\Delta}\right)^{2}(85)$

This can be written as, 
$\left(\frac{U}{\pi \Delta}\right)\left[\frac{1}{1+\left(\frac{\epsilon_{d}+U n_{c}}{\Delta}\right)^{2}}\right]=1$

Now, from (58), we have

$\rho_{d}(\epsilon=0)=\rho_{d}\left(\epsilon_{F}\right)=\left(\frac{1}{\pi \Delta}\right)\left[\frac{1}{1+\left(\frac{\epsilon_{d}+U n_{c}}{\Delta}\right)^{2}}\right]$

Using (87) in (86), we get

$U \rho_{d}\left(\epsilon_{F}\right)=1$

This is the necessary condition for magnetic stability.

The pioneering experimental works which, for the first time, revealed the presence of localized magnetic moments were performed by Matthias et al.[3] and then Wang et al. [20]. The results are reproduced in fig.4 and fig.5

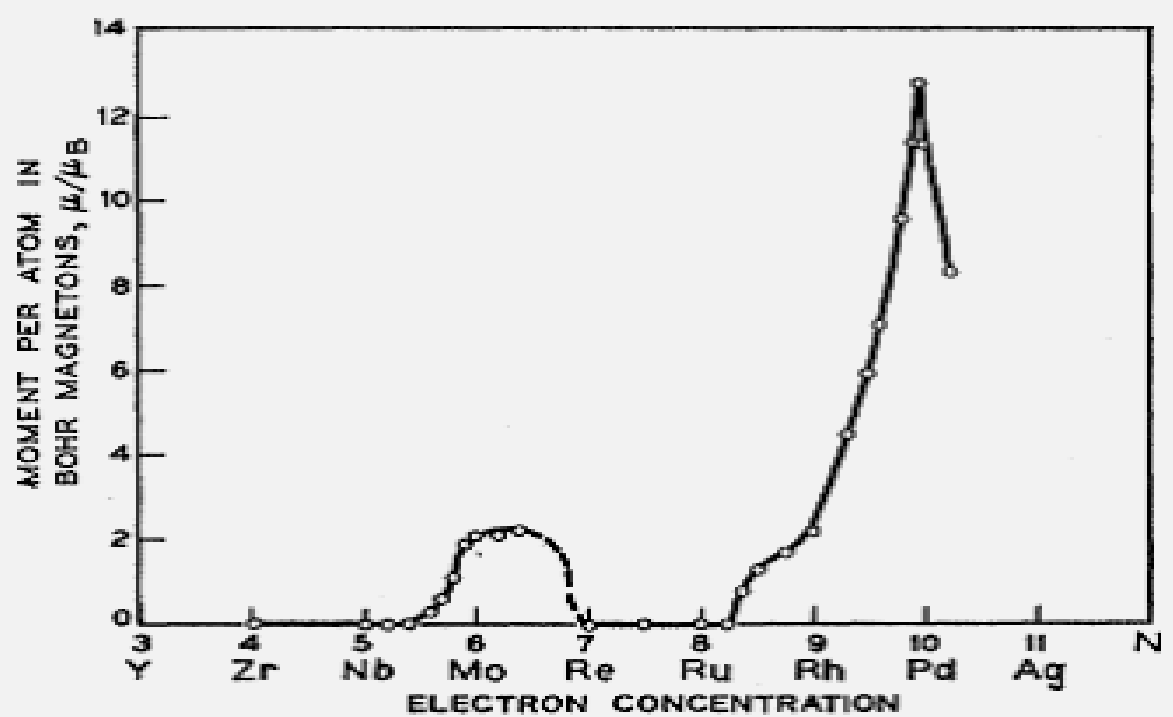

Fig.4 Magnetic moment in Bohr Magnetons of an iron atom dissolved in various second row transition metals (except Re which is a 5d element) and alloys as a function of electron concentration (Matthiaset al., 1962).

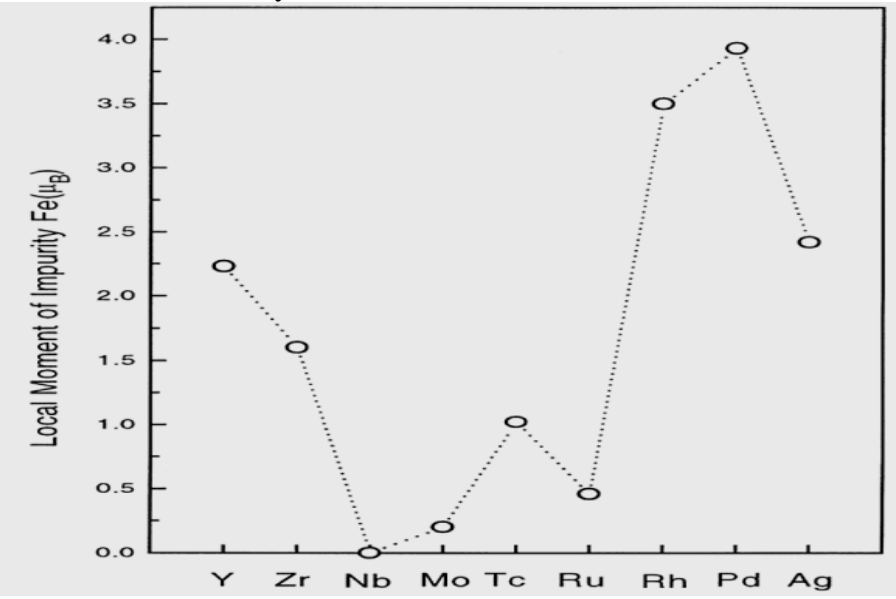

Fig.5. the variations of local magnetic moments of Fe with the 4d series (Wang et al., 1998)

From the above figures, it is clear that there are only selected alloys or metals with iron impurities, which exhibits presence of localized magnetic moments of varying magnitudes. This is in qualitative agreement with our theoretical results that magnetic moments cannot exist in all cases, but only in those cases, where the parameters $\epsilon_{d}, \Delta$ and $\mathrm{U}$ satisfy certain specified conditions separating magnetic and non-magnetic phases.

IV.

CONCLUSION

For a long time it has been achallenging problem for the theoretical physicists to comprehend the complex physics involved in the formation of magnetic moments in non-magnetic alloys and metals. In this context, the schematic model based on an effective Hamiltonian, presented in this paper, proves to be quite successful in the 
understanding of the complexities of the physical mechanisms responsible for the formation of persistent magnetic moments in non-magnetic host metals or alloys. A detailed microscopic calculation of the impurity energy, the on-site repulsion energy and the hybridization energy is highly formidable and hence a one -to-one quantitative comparison between theory and experiment is rather impossible. In this respect, the qualitative agreement of theory with experiment, shown in this work, is really encouraging.

\section{REFERENCES}

[1] W. De Haas,J. De Beor and G.J Van Den Berg, physica, Vol.1, pp1115, 1934.

[2] J. Kondo,Progr. Theoret. Phys. (kyoto), Vol.28, pp846, 1962; Vol.32, pp37, 1964

[3] B.T. Matthias, M. Peter, H. J. Williams, A.M. Clogston, E. Corenzwit, and R.C. Sherwood,phys. Rew.lett.,Vol.125, pp 541, 1962

[4] D. Riegel and K.D. Gross, Phys. Rev. B, vol163, pp678, 1990.

[5] J. Kappor, A.Metx, D. Riegal, R.Zeller, K.D. Gross, P.Schwalback, M.Eritic, E. Kankeleit, and W.D. Brewer, Europhys. Lett., Vol. 24, pp 299, 1993.

[6] A. Weismann, M. Wenderoth, S.Lounis, P.Zahu, N. Wuaas, R.G. Ulbrich, P.H. Dederichs, and S.Blugel, S.Scionce, vol. 323, pp. 1190, 2009,

[7] P.W. Anderson, Phys. Rev., VOl 124, pp 41, (1961).

[8] J.Friedel, NuovoCimento Suppl., Vol. 7, p.p 287, 1958.

[9] A komink and A.O. Gogolin, arxiv: cond-mat/0402469, 2008

[10] Chunhua fi, Jian-Xin Zhu and C.S.Ting, arxiv: cond-mat/1106.5827, 2011

[11] B. Uchoa, V.N. Kotov, N.M.R. Peres, and A.H. Castro Neto, Phys. Rev. Lett., Vol. 101, 026805, 2008

[12] H.B. Zhuang, Q.F. Sun and X.C. Xie, Europhys. Lett., Vol. 86, 58004, 2009

[13] D.Jacob and G. Kotliar, Phys. Rev. B, Vol. 82, 085423, 2010

[14] M.Vojta, L. Fritz, and R. Bulla, Europhys. Lett. Vol. 90, 27006, 2010.

[15] Gerd Bergmann, Phys. Rev. B, Vol. 73, 092418, 2006

[16] G. Meschede, F.steglich, W. Felsch, H. NAletta, and W. Zinn, Phys. Rev. Lett., Vol. 44, p.102, 1980.

[17] R. Siemann and B.R. Cooper, Phys. Rev., Vol. 44, p1015, 1980

[18] D.N. Zubarev, Siviet physics Upsekhi, Vol. 3, and pp.3 1960

[19] S.N. Biswas, Quantum Mechanics, Book abd Allied (p) Let, Calentta, 1998, pp65

[20] Q. Wang, Q. Suw, J.Z. yu, Zeng, and y. kawazoe, Journal of Magnetism and Magnetic Materials, Vol. 184, 1998. 